\title{
The Mini-EUSO telescope on board the International Space Station: Launch and first results
}

\begin{abstract}
M Casolino, ${ }^{a, f, g, *}$ D Barghini, ${ }^{b, c}$ M Battisti, ${ }^{b, c}$ A Belov, ${ }^{e}$ M Bertaina, ${ }^{b, c}$ F Bisconti, ${ }^{b, c}$ C Blaksley, ${ }^{f}$ K Bolmgren, ${ }^{h}$ F Cafagna, ${ }^{p}$ G Cambiè, ${ }^{b, g}$ F Capel, ${ }^{n}$ T Ebisuzaki, ${ }^{f}$ F Fenu,,${ }^{b, c}$ A Franceschi, ${ }^{j}$ C Fuglesang, ${ }^{h}$ A Golzio, ${ }^{b, c} \mathbf{P}$ Gorodetzki, ${ }^{f}$ F Kajino, ${ }^{m}$ H Kasuga, ${ }^{f} \mathbf{P}$ Klimov, ${ }^{e}$ V. Kungel, ${ }^{r} \mathbf{M}$ Manfrin, ${ }^{b, c} \mathbf{L}$ Marcelli, ${ }^{a}$ W Marszał, ${ }^{k} \mathbf{H}$ Miyamoto, ${ }^{b, c} \mathbf{M}$ Mignone, ${ }^{b, c}$ T Napolitano, ${ }^{j}$ G Osteria, ${ }^{q}$ E Parizot, ${ }^{l}$ P Picozza, ${ }^{a, g}$ L W Piotrowski, ${ }^{o} \mathbf{Z}$ Plebaniak, ${ }^{b}$ G Prévôt, ${ }^{l}$ E Reali, ${ }^{a, g}$ M Ricci, ${ }^{j}$ N Sakaki, ${ }^{f}$ K Shinozaki, ${ }^{k}$ J Szabelski, ${ }^{k}$ Y Takizawa, ${ }^{f} \mathbf{S}$ Wada $^{f}$ and L. Wiencke ${ }^{r}$ on behalf of the JEM-EUSO Collaboration
\end{abstract} (a complete list of authors can be found at the end of the proceedings)

${ }^{a}$ INFN, Sezione di Roma Tor Vergata - Roma, Italy

${ }^{b}$ INFN, Sezione di Torino - Torino, Italy

${ }^{c}$ Dipartimento di Fisica, Universitá di Torino, Italy

${ }^{d}$ Faculty of Physics, M.V. Lomonosov Moscow State University - Moscow, Russia

e Skobeltsyn Institute of Nuclear Physics, Lomonosov Moscow State Univ. - Moscow, Russia

${ }^{f}$ RIKEN - Wako, Japan

${ }^{g}$ Universitá degli Studi di Roma Tor Vergata - Dipartimento di Fisica, Roma, Italy

${ }^{h}$ KTH Royal Institute of Technology - Stockholm, Sweden

${ }^{i}$ Université de Paris, CNRS, Astroparticule et Cosmologie, F-75006 Paris, France

jINFN-LNF - Frascati, Italy

${ }^{k}$ National Centre for Nuclear Research - Lodz, Poland

${ }^{l}$ APC, Univ Paris Diderot, CNRS/IN2P3, CEA/Irfu, Obs de Paris, Sorbonne Paris Cité, France

${ }^{m}$ Konan University, Kobe, Japan

${ }^{n}$ Technical University of Munich- Munich, Germany

${ }^{o}$ Faculty of Physics, University of Warsaw - Warsaw, Poland

${ }^{p}$ INFN, Sezione di Bari - Bari, Italy

${ }^{q}$ INFN, Sezione di Napoli - Napoli, Italy

${ }^{r}$ Colorado School of Mines, Golden, CO, USA

E-mail: casolino@roma2.infn.it

\footnotetext{
${ }^{*}$ Presenter
} 
Mini-EUSO is a telescope launched on board the International Space Station in 2019 and currently located in the Russian section of the station. Main scientific objectives of the mission are the search for nuclearites and Strange Quark Matter, the study of atmospheric phenomena such as Transient Luminous Events, meteors and meteoroids, the observation of sea bioluminescence and of artificial satellites and man-made space debris. It is also capable of observing Extensive Air Showers generated by Ultra-High Energy Cosmic Rays with an energy above $10^{21} \mathrm{eV}$ and detect artificial showers generated with lasers from the ground. Mini-EUSO can map the night-time Earth in the UV range $(290-430 \mathrm{~nm})$, with a spatial resolution of about $6.3 \mathrm{~km}$ and a temporal resolution of $2.5 \mu \mathrm{s}$, observing our planet through a nadir-facing UV-transparent window in the Russian Zvezda module. The instrument, launched on 2019/08/22 from the Baikonur cosmodrome, is based on an optical system employing two Fresnel lenses and a focal surface composed of 36 Multi-Anode Photomultiplier tubes, 64 channels each, for a total of 2304 channels with single photon counting sensitivity and an overall field of view of $44^{\circ}$. Mini-EUSO also contains two ancillary cameras to complement measurements in the near infrared and visible ranges. In this paper we describe the detector and present the various phenomena observed in the first year of operation.

$37^{\text {th }}$ International Cosmic Ray Conference (ICRC 2021)

July 12th - 23rd, 2021

Online - Berlin, Germany 


\section{Introduction}

Mini-EUSO (Multiwavelength Imaging New Instrument for the Extreme Universe Space Observatory or 'UV atmosphere' as is known in the Russian Space Program) is a telescope operating in the UV range $(290-430 \mathrm{~nm})$ with a square field of view of $\simeq 44^{\circ}$ and a ground resolution of $\simeq 6.3 \times 6.3 \mathrm{~km}^{2}$, depending on the altitude of the International Space Station (ISS). Mini-EUSO was brought to the ISS by the uncrewed Soyuz MS-14, on 2019/08/22. First observations from the nadir-facing UV transparent window in the Russian Zvezda module took place on October 7. Since then, it has been taking data periodically, with installations occurring every couple of weeks. The instrument is expected to operate for at least three years. For more details on the detector and the data gathered so far see [1].

The optical system consists of two Fresnel lenses with a diameter of $25 \mathrm{~cm}$. The focal surface, or Photon Detector Module (PDM), consists of 36 MultiAnode Photomultipliers (MAPMTs) tubes by Hamamatsu, 64 pixels each, capable of single photon detection. Readout is handled by ASICs (Application Specific Integrated Circuit) in frames of $2.5 \mu s$ (1 Gate Time Unit, GTU). Data are then processed by a Zynq based FPGA board which implements a multi-level triggering, allowing the measurement of triggered UV transients for 128 frames at time scales of both $2.5 \mu \mathrm{s}$ and $320 \mu \mathrm{s}$ (see [5] for a description of the trigger system and its performance). A continuous acquisition mode with $\simeq 40.96 \mathrm{~ms}$ frames is also performed.

\section{Instrument Overview}

Mini-EUSO has been designed to be installed in the interior of the ISS on the UV-transparent window located in the Zvezda module 1). The dimensions $\left(37 \times 37 \times 62 \mathrm{~cm}^{3}\right)$ are thus defined by the size of the window and the constraints of the Soyuz spacecraft. Furthermore, the design accommodates the requirements of safety (no sharp edges, low surface temperature, robustness...) to the crew. Coupling to the window is done via a mechanical adapter flange; the only connection to the ISS is via a $28 \mathrm{~V}$ power supply and grounding cable. The power consumption of the telescope is $\simeq 60 \mathrm{~W}$ and the weight is $35 \mathrm{~kg}$, including the $5 \mathrm{~kg}$ flange. For each observation session, taking place about every two weeks and of the duration of about 12 hours, the instrument is removed from storage and installed on the UV window. Data are stored on 512 GB USB Solid State Disks (SSD) that are inserted in the side of the telescope by the astronauts. No direct telecommunication with ground is present, but samples of data (about 10\%, usually corresponding to the beginning and the end of each session) from each session are copied by the crew and transmitted to ground to verify the correct functioning of the instrument and optimize its working parameters. Conversely, before each session, working parameters, patches in software and hardware are uplinked to the ISS and then copied on the SSD disk to fine-tune the acquisition of the telescope. Pouches with 25 SSDs are returned to Earth every 6 months.

The optics consists of two, $25 \mathrm{~cm}$ diameter, Fresnel lenses with a wide field of view ( $44^{\circ}$ seen from the PDM). Poly(methyl methacrylate) - PMMA - is used to manufacture the lenses with a diamond bit machine. In this way it is possible to have a light (11 mm thickness, $0.87 \mathrm{~kg} / \mathrm{lens}$ ), robust and compact design well suited for space applications. The effective focal length of the 
system is $300 \mathrm{~mm}$, with a Point Spread Function (PSF) of 1.2 pixels, of the same dimension as the pixel size of the MAPMTs.

The Mini-EUSO focal surface (PDM) consists of a matrix of 36 Multi-Anode Photomultiplier Tubes (MAPMTs, Hamamatsu Photonics R11265-M64), arranged in an array of $6 \times 6$ elements. Each MAPMT consists of $8 \times 8$ pixels, resulting in a total of 2304 channels. The MAPMTs are grouped in Elementary Cells (ECs), each with $2 \times 2$ units. Each of the nine ECs of the PDM shares a common high voltage power supply and a board connecting the dynodes and anodes of the four photomultipliers. The whole system ( $250 \mathrm{~g}$ each EC, including filters and MAPMTs) is potted with Arathane and located in the shadow of the photosensors.

After the integration and acceptance tests [2,3], first in Rome, subsequently in Moscow and finally in Baikonur cosmodrome, the detector was integrated in the uncrewed Soyuz capsule and launched on 2019/08/22. The telescope was first turned on 2019/10/7 (Figure 2). The first session involved operation in safe mode, with only one EC unit active and the HVPS set to last dynode voltage mode, corresponding to a sensitivity of about $1 \%$ compared to the normal HVPS mode. Gradually, along the course of the following sessions, the subsequent acquisitions have used the full PDM in normal voltage mode. See [4] for a description of the software and the acquisition procedure.

\section{Observations}

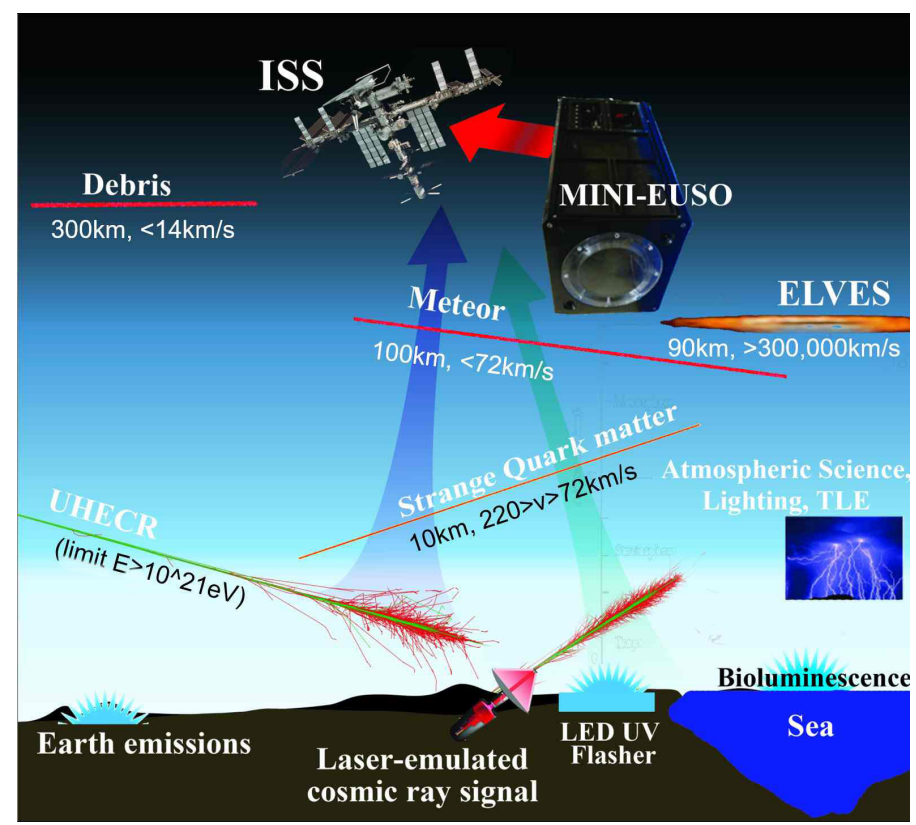

Figure 1: Main science goals of Mini-EUSO. Thanks to its various time acquisitions, ranging from $2.5 \mu \mathrm{s}$ to $40 \mathrm{~ms}$, the telescope is capable of addressing phenomena with various duration, from the slow terrestrial emissions (minutes, seconds) to Transient Luminous Events such as ELVEs $(\simeq 300-400 \mu s)$.

Mini-EUSO is capable of observations (Figure 1) of various phenomena occurring at various time scales. Figure 3 shows the observed total signal of the focal surface as a function of time 


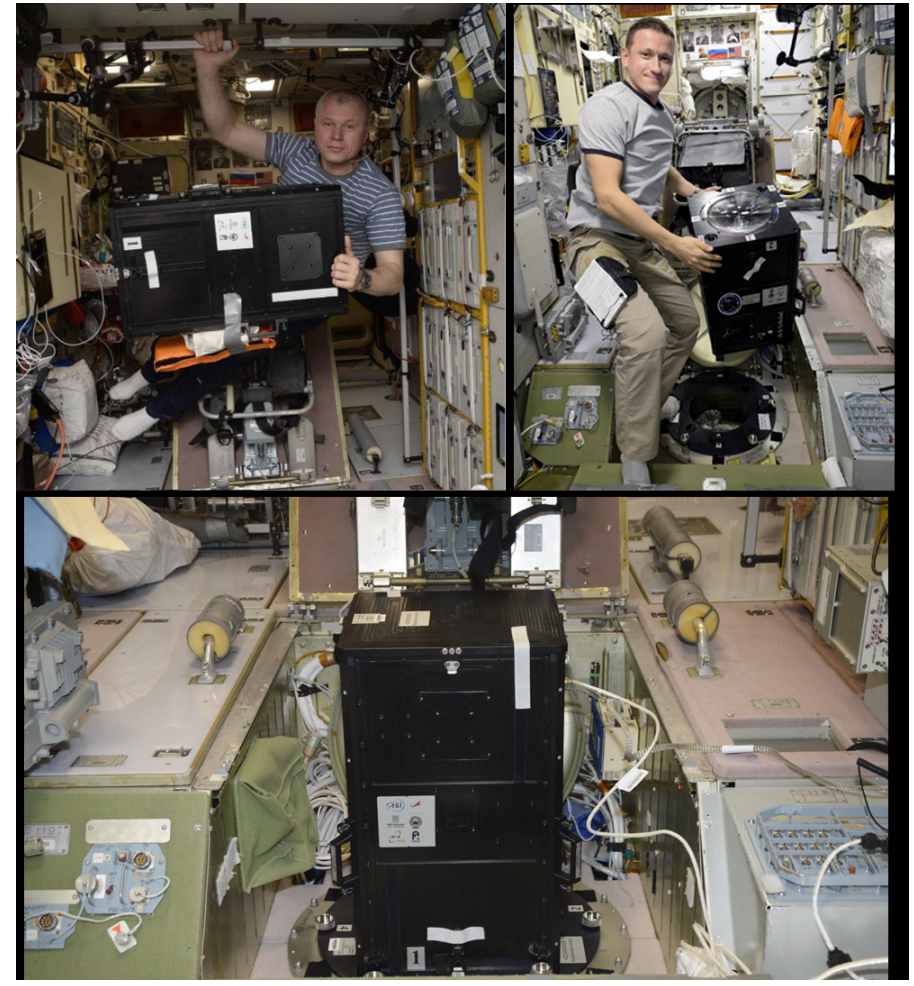

Figure 2: Top left and right: Cosmonaut Oleg Novitsky and Sergey Kud-Sverchkov installing Mini-EUSO in the Zvezda module. Bottom: Mini-EUSO installed on the UV transparent window of the module.

for events from the faster $2.5 \mu$ s sampling (D1) to the 128 frame averages for D2 (320 $\mu$ s) to the $128 \times 128$ frame average for D3 (40.96 ms). In the longer time frames, the gradual increase is due to the passage over a clouded area, whereas the sharp spikes are due to lightning. Large lightning triggers the safety system of the detector, resulting in the temporary deactivation of the HVPS of the EC unit which would be overexposed by lightning.

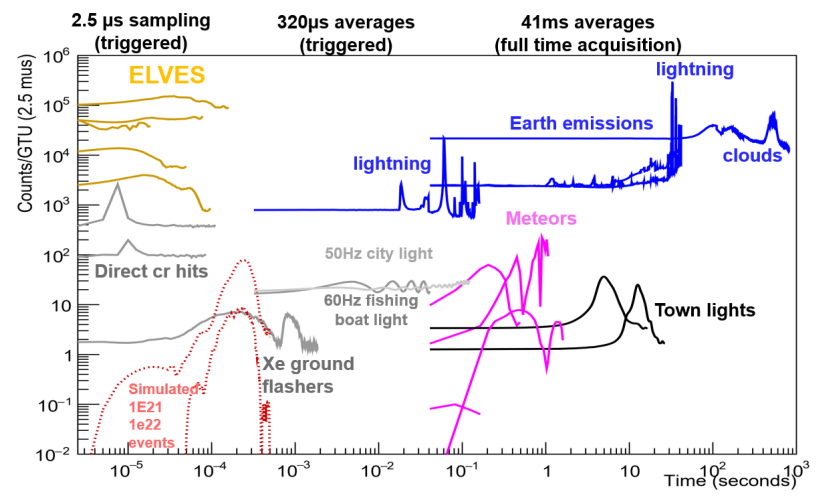

Figure 3: Temporal profile of various signals observed by Mini-EUSO. All plots refer to data acquired on the ISS (except the simulations of $10^{21}$ and $10^{22} \mathrm{eV}$ UHECRs).

The main observations possible by Mini-EUSO are:

1. Night UV emissions from the Earth. Mini-EUSO can map the Earth in the near UV range 


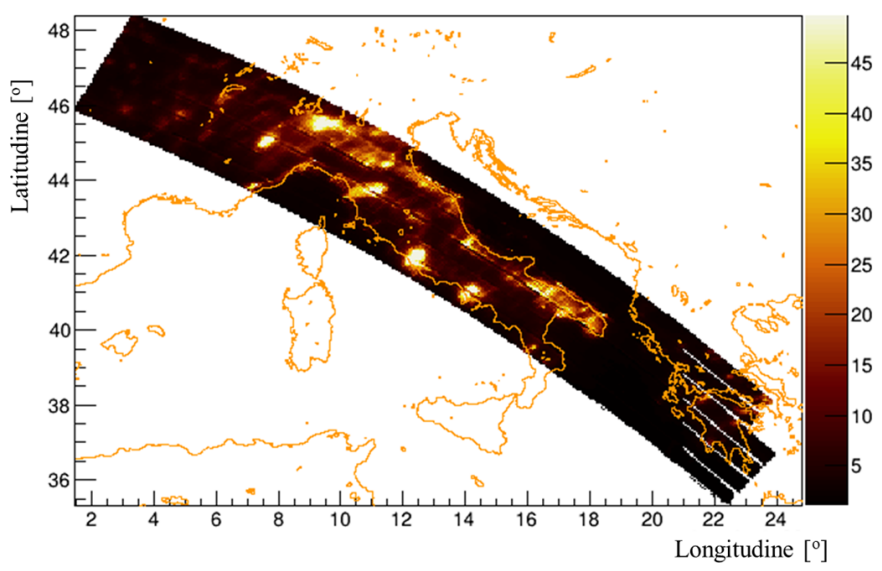

Figure 4: Observation of night time emissions from Italy. The light from the various lights can be distinguished, as well as the different emissions from ground and sea.

with spatial and temporal resolution of $\sim 6.3 \times 6.3 \mathrm{~km}^{2}$ and $2.5 \mu$ s respectively, measuring variations of the UV emissions. See Figure 4 for an example of the mapping capabilities of the instrument $[6,7]$. Several campaigns with ground LED flashers have been carried out [5] or will be carried out with LED and lasers shooting in the field of view (f.o.v.) of Mini-EUSO.

2. Space debris. Attempts will be made to track space debris to investigate the possibility of using laser ablation for their removal. The maximum detection distance of Mini-EUSO is about $100 \mathrm{~km}$ for debris size of $0.1 \mathrm{~m}$. This observation is restricted to the local twilight period of the orbit, about 5 min every $90 \mathrm{~min}$ [8].

3. Meteors are relatively slow ( $v \leq 72 \mathrm{~km} / \mathrm{s}$ ) and long-lasting (a few seconds) events which illuminate in sequence several light sensitive pixels of the Mini-EUSO focal surface (See Figure 5 for the reconstruction of a typical meteor event). So far we have observed more than 5000 meteor events. The maximum observable magnitude is between 5 and 6 depending on background conditions $[9,10]$. The frequency-intensity distribution of the observation of meteors will allow to make an inventory of the population of near-Earth objects from space with a large field of view and the advantage of not being covered by clouds. Events coming from interstellar meteors (with a kinetic energy above $72 \mathrm{~km} / \mathrm{s}$ ) and Strange Quark Matter (SQM) candidates (appearing as long-lasting, constant-luminosity events) can also be observed [11].

4. Transient Luminous Events, in particular ELVES are observed as large ring-like upper atmospheric emissions that appear to be expanding at superluminal speed[12]. In Figure 6 are shown the pictures of one ELVE (observed on 2020/05/26) entering the field of view. Furthermore, it will be possible to make joint observations with other detectors on board the ISS such as Altea-Lidal and ASIM.

5. UHECR. Mini-EUSO can measure fluorescence and Cherenkov light emitted by UHECR initiated showers. The diameter of the lens system - constrained by the size of the ISS 
window - places the threshold energy for UHECR detection around $10^{21} \mathrm{eV}$. We estimate an yearly exposure - after commissioning and selecting observations close to new moon and in regions of low background - to be of the order of $1000 \mathrm{~km}^{2} \mathrm{sr}$ yr. The absence of events with energy above $3 \cdot 10^{20} \mathrm{eV}$ obtained with ground detectors make it unlikely for Mini-EUSO to observe any event at these energies[13]. We note that Mini-EUSO exposure is of the same order of magnitude of the fluorescence detector of Telescope Array, so it can contribute to search for exotic events that would not give a signal in the surface detectors.
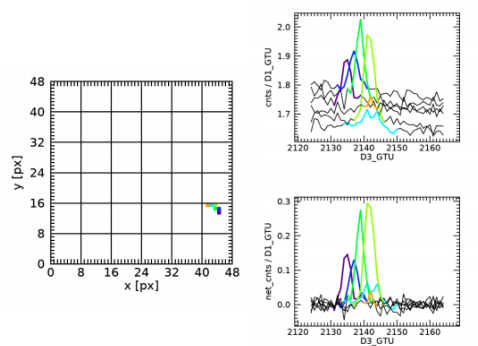
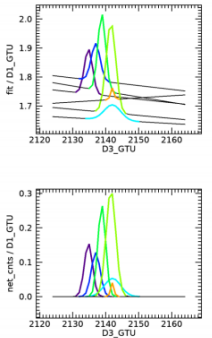
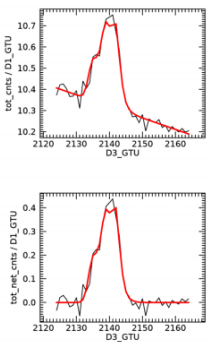

Figure 5: Left: A meteor track as it develops in the field of view of Mini-EUSO. The $X$ and Y axis represent the PDM pixels. Right top: the meteor track counts of each pixel, the resulting light curve and, Right bottom: the same curves with background subtracted [14].

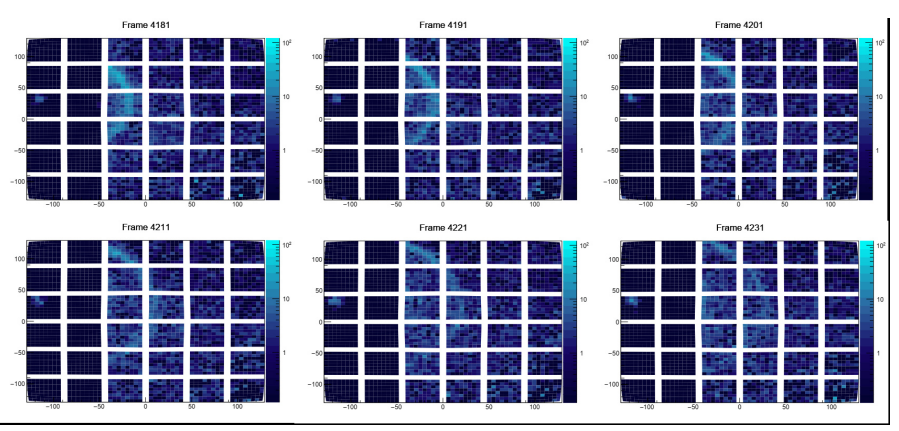

Figure 6: A sample of frames of an ELVE being observed in the focal surface. The left column of three EC units is temporarily working at a $1 / 1000$ sensitivity due to a previous bright light that triggered the safety mechanism. This allows to see the lightning in the centre left of the focal surface, which generates the expanding elve ring in the centre of the FS. Pictures are 10 frames apart, thus $2.5 \times 10=25 \mu$ s apart.

\section{Conclusions}

Initial analysis of the Mini-Euso data received in the first year of operations confirms the correct functioning of the instrument. We have observed events in all the operational time frames, from the fast ELVEs, to meteors (40.96 ms readout), lighting and terrestrial emissions.

\section{Acknowledgments}

This work was partially supported by Basic Science Interdisciplinary Research Projects of RIKEN and JSPS KAKENHI Grant (22340063, 23340081, and 24244042), by the Italian Ministry of Foreign Affairs and International Cooperation, by the Italian Space Agency through the 
ASI INFN agreements n. 2017-8-H.0 and n. 2021-8-HH.0, by NASA award 11-APRA-0058, 16-APROBES16-0023, 17-APRA17-0066, NNX17AJ82G, NNX13AH54G, 80NSSC18K0246, 80NSSC18K0473, 80NSSC19K0626, and 80NSSC18K0464 in the USA, by the French space agency CNES, by the Deutsches Zentrum für Luft- und Raumfahrt, the Helmholtz Alliance for Astroparticle Physics funded by the Initiative and Networking Fund of the Helmholtz Association (Germany), by Slovak Academy of Sciences MVTS JEM-EUSO, by National Science Centre in Poland grants 2017/27/B/ST9/02162 and 2020/37/B/ST9/01821, by Deutsche Forschungsgemeinschaft (DFG, German Research Foundation) under Germany's Excellence Strategy - EXC-2094390783311, by Mexican funding agencies PAPIIT-UNAM, CONACyT and the Mexican Space Agency (AEM), as well as VEGA grant agency project 2/0132/17, and by by State Space Corporation ROSCOSMOS and the Interdisciplinary Scientific and Educational School of Moscow University "Fundamental and Applied Space Research".

\section{References}

[1] S. Bacholle et al, ApJ Supp., 253, 2, 36, 17, 2020.

[2] G. Cambiè et al (JEM-EUSO Coll.), Integration and qualification of the Mini-EUSO telescope on board the ISS, PoS(ICRC2021) 1001.

[3] Belov, A., Cambiè, G., Casolino, M., et al. 2020, Aerotecnica Missili e Spazio, 99, 93, 10.1007/s42496-020-00047-1.

[4] F. Capel et al, JATIS, 5(4), 044009 (2019).

[5] M. Battisti et al (JEM-EUSO Coll.), Overview of the Mini-EUSO $\mu$ s trigger logic performance, PoS(ICRC2021) 411.

[6] A. Golzio et al (JEM-EUSO Coll.), A study on UV emission from clouds with Mini-EUSO, PoS(ICRC2021) 417

[7] K. Shinozaki et al, PoS(ICRC2021) 1165.

[8] Ebisuzaki, T., et al. Acta Astronautica, 112, 102, 2016

[9] Abdellaoui, G., et al., 2017, Planetary and Space Science, 143, 245, 10.1016/j.pss.2016.12.001

[10] Adams, J, et al, Experimental Astronomy, 40, 253, 10.1007/s10686-014-9375-4, 2015.

[11] L. Piotrowski et al (JEM-EUSO Coll.), Towards observations of nuclearites with Mini-EUSO, PoS(ICRC2021) 1181.

[12] L. Marcelli et al (JEM-EUSO Coll.), Observations of Transient Luminous Events with the Mini-EUSO telescope on board the ISS, PoS(ICRC2021) 971.

[13] F. Fenu et al (JEM-EUSO Coll.), Simulation studies for the Mini-EUSO detector, PoS(ICRC2021) 757.

[14] D. Barghini, 14th Europlanet Science Congress 2020, EPSC2020-800 September 2020 2020EPSC...14..800B 


\section{The JEM-EUSO Collaboration}

(author-list as of July 1st, 2021)

G. Abdellaoui ${ }^{a h}$, S. Abe ${ }^{f q}$, J.H. Adams Jr. ${ }^{p d}$, D. Allard ${ }^{c b}$, G. Alonso ${ }^{m d}$, L. Anchordoqui ${ }^{p e}$, A. Anzalone ${ }^{e h, e d}$, E. Arnone ${ }^{e k, e l}$, K. Asano ${ }^{f e}$, R. Attallah ${ }^{a c}$, H. Attoui $^{a a}$, M. Ave Pernas ${ }^{m c}$, M. Bagheri $^{p h}$, J. Baláz ${ }^{l a}$, M. Bakiri ${ }^{a a}$, D. Barghiniel ${ }^{e l e k}$, S. Bartocci ${ }^{e i, e j}$, M. Battisti ${ }^{e k, e l}$, J. Bayer ${ }^{d d}$, B. Beldjilali ${ }^{a h}$, T. Belenguer ${ }^{m b}$, N. Belkhalfa ${ }^{a a}$, R. Bellotti ${ }^{e a, e b}$, A.A. Belov ${ }^{k b}$, K. Benmessai ${ }^{a a}$, M. Bertaina ${ }^{e k, e l}$, P.F. Bertone ${ }^{p f}$, P.L. Biermann ${ }^{d b}$, F. Bisconti ${ }^{e l, e k}$, C. Blaksley ${ }^{f t}$, N. Blanc ${ }^{o a}$, S. Blin-Bondil ${ }^{c a, c b}$, P. Bobik ${ }^{l a}$, M. Bogomilov ${ }^{b a}$, E. Bozzo ${ }^{o b}$, S. Briz ${ }^{p b}$, A. Bruno ${ }^{e h, e d}$, K.S. Caballero ${ }^{h d}$, F. Cafagna ${ }^{e a}$, G. Cambié $^{e i, e j}$, D. Campana ${ }^{e f}$, J-N. Capdevielle ${ }^{c b}$, F. Capel ${ }^{d e}$, A. Caramete $^{j a}$, L. Caramete $^{j a}$, P. Carlson $^{n a}$,

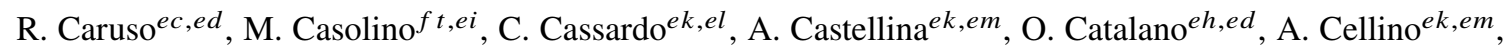
K. Černýb ${ }^{b b}$, M. Chikawa ${ }^{f c}$, G. Chiritoi ${ }^{j a}$, M.J. Christl ${ }^{p f}$, R. Colalillo ${ }^{e f, e g}$, L. Conti ${ }^{e n, e i}$, G. Cotto ${ }^{e k, e l}$, H.J. Crawford ${ }^{p a}$, R. Cremonini ${ }^{e l}$, A. Creusot ${ }^{c b}$, A. de Castro Gónzalez ${ }^{p b}$, C. de la Taille ${ }^{c a}$, L. del Peral $^{m c}$, A. Diaz Damian ${ }^{c c}$, R. Diesing ${ }^{p b}$, P. Dinaucourt ${ }^{c a}$, A. Djakonow $^{i a}$, T. Djemil ${ }^{a c}$, A. Ebersoldt ${ }^{d b}$, T. Ebisuzaki ${ }^{f t}$, L. Eliasson ${ }^{n a}$, J. Eser ${ }^{p b}$, F. Fenu ${ }^{e k, e l}$, S. Fernández-González ${ }^{m a}$, S. Ferrarese ${ }^{e k, e l}$, G. Filippatos ${ }^{p c}$, W.I. Finch ${ }^{p c}$ C. Fornaro ${ }^{e n, e i}$, M. Fouka ${ }^{a b}$, A. Franceschi ${ }^{e e}$, S. Franchini ${ }^{m d}$, C. Fuglesang ${ }^{n a}$, T. Fujii ${ }^{f g}$, M. Fukushima ${ }^{f e}$, P. Galeotti ${ }^{e k, e l}$, E. García-Ortega ${ }^{m a}$, D. Gardiolelem, G.K. Garipov ${ }^{k b}$, E. Gascón ${ }^{m a}$, E. Gazda ${ }^{p h}$, J. Gencil ${ }^{l b}$, A. Golzio ${ }^{e k, e l}$, C. González Alvarado ${ }^{m b}$, P. Gorodetzky ${ }^{f t}$, A. Green ${ }^{p c}$, F. Guarino ${ }^{e f, e g}$, C. Guépin ${ }^{p l}$, A. Guzmán ${ }^{d d}$, Y. Hachisu ${ }^{f t}$, A. Haungs ${ }^{d b}$, J. Hernández

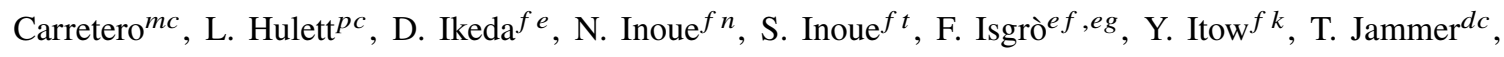
S. Jeong ${ }^{g b}$, E. Joven ${ }^{m e}$, E.G. Judd ${ }^{p a}$, J. Jochum ${ }^{d c}$, F. Kajino ${ }^{f f}$, T. Kajino ${ }^{f i}$, S. Kalli ${ }^{a f}$, I. Kaneko ${ }^{f t}$, Y. Karadzhov ${ }^{b a}$, M. Kasztelan ${ }^{i a}$, K. Katahira ${ }^{f t}$, K. Kawai ${ }^{f t}$, Y. Kawasaki ${ }^{f t}$, A. Kedadra ${ }^{a a}$, H. Khales ${ }^{a a}$, B.A. Khrenov ${ }^{k b}$, Jeong-Sook Kim ${ }^{g a}$, Soon-Wook Kim ${ }^{g a}$, M. Kleifges ${ }^{d b}$, P.A. Klimov ${ }^{k b}$, D. Kolev ${ }^{b a}$, I. Kreykenbohm ${ }^{d a}$, J.F. Krizmanic ${ }^{p f, p k}$, K. Królik ${ }^{i a}$, V. Kungel ${ }^{p c}$, Y. Kurihara ${ }^{f s}$, A. Kusenko ${ }^{f r}, p e$, E. Kuznetsov ${ }^{p d}$, H. Lahmar ${ }^{a a}$, F. Lakhdari ${ }^{a g}$, J. Licandro ${ }^{m e}$, L. López Campano ${ }^{m a}$, F. López Martínez ${ }^{p b}$, S. Mackovjak ${ }^{l a}$, M. Mahdi ${ }^{a a}$, D. Mandát ${ }^{b c}$, M. Manfrin ${ }^{e k, e l}$, L. Marcellie ${ }^{e i}$, J.L. Marcos ${ }^{m a}$, W. Marszał ${ }^{i a}$, Y. Martín ${ }^{m e}$, O. Martinez ${ }^{h c}$, K. Mase ${ }^{f a}$, R. Matev ${ }^{b a}$, J.N. Matthews ${ }^{p g}$, N. Mebarki ${ }^{a d}$, G. MedinaTanco $^{h a}$, A. Menshikov ${ }^{d b}$, A. Merino ${ }^{m a}$, M. Mese ${ }^{e f, e g}$, J. Meseguer $^{m d}$, S.S. Meyer ${ }^{p b}$, J. Mimouni ${ }^{a d}$, H. Miyamoto ${ }^{e k, e l}$, Y. Mizumoto ${ }^{f i}$, A. Monaco ${ }^{e a, e b}$, J.A. Morales de los Ríos ${ }^{m c}$, M. Mastafa ${ }^{p d}$, S. Nagataki ${ }^{f t}$, S. Naitamor ${ }^{a b}$, T. Napolitano ${ }^{e e}$, J. M. Nachtman ${ }^{p i}$ A. Neronov ${ }^{o b, c b}$, K. Nomoto ${ }^{f r}$, T. Nonaka ${ }^{f e}$, T. Ogawa ${ }^{f t}$, S. Ogio ${ }^{f l}$, H. Ohmori ${ }^{f t}$, A.V. Olinto ${ }^{p b}$, Y. Onel ${ }^{p i}$ G. Osteria $^{e f}$, A.N. Otte ${ }^{p h}$, A. Pagliaro ${ }^{e h, e d}$, W. Painter ${ }^{d b}$, M.I. Panasyuk ${ }^{k b}$, B. Panico ${ }^{e f}$, E. Parizot ${ }^{c b}$, I.H. Park ${ }^{g b}$, B. Pastircak ${ }^{l a}$, T. Paul ${ }^{p e}$, M. Pech ${ }^{b b}$, I. Pérez-Grande ${ }^{m d}$, F. Perfetto ${ }^{e f}$, T. Peter ${ }^{o c}$, P. Picozza $^{e i, e j, f t}$, S. Pindado ${ }^{m d}$, L.W. Piotrowski ${ }^{i b}$, S. Piraino ${ }^{d d}$, Z. Plebaniak ${ }^{e k, e l, i a}$, A. Pollini ${ }^{o a}$, E.M. Popescu ${ }^{j a}$, R. Prevete ${ }^{e f, e g}$, G. Prévôt ${ }^{c b}$, H. Prieto ${ }^{m c}$, M. Przybylak ${ }^{i a}$, G. Puehlhofer ${ }^{d d}$, M. Putis ${ }^{l a}$, P. Reardon ${ }^{p d}$, M.H.. Reno ${ }^{p i}$, M. Reyes ${ }^{m e}$, M. Ricci ${ }^{e e}$, M.D. Rodríguez Frías ${ }^{m c}$, O.F. Romero Matamala ${ }^{p h}$, F. Ronga ${ }^{e e}$, M.D. Sabau ${ }^{m b}$, G. Saccáa ${ }^{e c, e d}$, G. Sáez Cano ${ }^{m c}$, H. Sagawa ${ }^{f e}$, Z. Sahnoune ${ }^{a b}$, A. Saito ${ }^{f g}$, N. Sakaki ${ }^{f t}$, H. Salazar ${ }^{h c}$, J.C. Sanchez Balanzar ${ }^{h a}$, J.L. Sánchez ${ }^{m a}$, A. Santangelo ${ }^{d d}$, A. Sanz-Andrés ${ }^{m d}$, M. Sanz Palomino ${ }^{m b}$, O.A. Saprykin ${ }^{k c}$, F. Sarazin ${ }^{p c}$, M. Sato ${ }^{f o}$, A. Scagliola ${ }^{e a, e b}$, T. Schanz ${ }^{d d}$, H. Schieler ${ }^{d b}$, P. Schovánek ${ }^{b c}$, V. Scotti ${ }^{f, e g}$, M. Serra ${ }^{m e}$, S.A. Sharakin ${ }^{k b}$, H.M. Shimizu ${ }^{f j}$, K. Shinozaki ${ }^{i a}$, T. Shirahama ${ }^{f n}$, J.F. Soriano ${ }^{p e}$, A. Sotgiu ${ }^{e i, e j}$, I. Stan ${ }^{j a}$, I. Strharskýla , N. Sugiyama ${ }^{f j}$, D. Supanitsky ${ }^{h a}$, M. Suzuki ${ }^{f m}$, J. Szabelski ${ }^{i a}$, N. Tajima ${ }^{f t}$, T. Tajima ${ }^{f t}$, Y. Takahashi ${ }^{f o}$, M. Takeda ${ }^{f e}$, Y. Takizawa ${ }^{f t}$, M.C. Talai ${ }^{a c}$, Y. Tameda ${ }^{f u}$, C. Tenzer ${ }^{d d}$, S.B. Thomas ${ }^{p g}$, O. Tibolla ${ }^{h e}$, L.G. Tkachev ${ }^{k a}$, T. Tomida ${ }^{f h}$, N. Tone ${ }^{f t}$, 


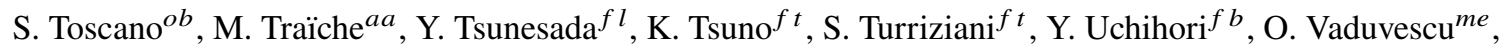
J.F. Valdés-Galicia ${ }^{h a}$, P. Vallania ${ }^{e k, e m}$, L. Valore ${ }^{e f, e g}$, G. Vankova-Kirilova ${ }^{b a}$, T. M. Venters ${ }^{p j}$, C. Vigorito ${ }^{e k, e l}$, L. Villaseñor ${ }^{h b}$, B. Vlcek ${ }^{m c}$, P. von Ballmoos ${ }^{c c}$, M. Vrabel ${ }^{l b}, \mathrm{~S}$. Wada ${ }^{f t}, \mathrm{~J}_{\text {. Watanabe }}^{f i}$, J. Watts Jr. ${ }^{p d}$, R. Weigand Muñoz ${ }^{m a}$, A. Weindl ${ }^{d b}$, L. Wiencke ${ }^{p c}$, M. Wille ${ }^{d a}$, J. Wilms ${ }^{d a}$, D. Winn ${ }^{p m}$ T. Yamamoto $f f$, J. Yang ${ }^{g b}$, H. Yano ${ }^{f m}$, I.V. Yashin ${ }^{k b}$, D. Yonetoku ${ }^{f d}$, S. Yoshida ${ }^{f a}$, R. Young ${ }^{p f}$, I.S Zgura ${ }^{j a}$, M.Yu. Zotov ${ }^{k b}$, A. Zuccaro Marchi ${ }^{f t, f u}$

aa Centre for Development of Advanced Technologies (CDTA), Algiers, Algeria

$a b$ Dep. Astronomy, Centre Res. Astronomy, Astrophysics and Geophysics (CRAAG), Algiers, Algeria

${ }^{a c}$ LPR at Dept. of Physics, Faculty of Sciences, University Badji Mokhtar, Annaba, Algeria

${ }^{a d}$ Lab. of Math. and Sub-Atomic Phys. (LPMPS), Univ. Constantine I, Constantine, Algeria

af Department of Physics, Faculty of Sciences, University of M'sila, M'sila, Algeria

ag Research Unit on Optics and Photonics, UROP-CDTA, Sétif, Algeria

ah Telecom Lab., Faculty of Technology, University Abou Bekr Belkaid, Tlemcen, Algeria

ba St. Kliment Ohridski University of Sofia, Bulgaria

$b b$ Joint Laboratory of Optics, Faculty of Science, Palacký University, Olomouc, Czech Republic

${ }^{b c}$ Institute of Physics of the Czech Academy of Sciences, Prague, Czech Republic

ca Omega, Ecole Polytechnique, CNRS/IN2P3, Palaiseau, France

${ }^{c b}$ Université Paris, CNRS, AstroParticule et Cosmologie, F-75013 Paris, France

$c c$ IRAP, Université de Toulouse, CNRS, Toulouse, France

$d a$ ECAP, University of Erlangen-Nuremberg, Germany

${ }^{d b}$ Karlsruhe Institute of Technology (KIT), Germany

${ }^{d c}$ Experimental Physics Institute, Kepler Center, University of Tübingen, Germany

$d d$ Institute for Astronomy and Astrophysics, Kepler Center, University of Tübingen, Germany

de Technical University of Munich, Munich, Germany

ea Istituto Nazionale di Fisica Nucleare - Sezione di Bari, Italy

${ }^{e b}$ Universita' degli Studi di Bari Aldo Moro and INFN - Sezione di Bari, Italy

ec Dipartimento di Fisica e Astronomia "Ettore Majorana", Universita' di Catania, Italy

ed Istituto Nazionale di Fisica Nucleare - Sezione di Catania, Italy

ee Istituto Nazionale di Fisica Nucleare - Laboratori Nazionali di Frascati, Italy

ef Istituto Nazionale di Fisica Nucleare - Sezione di Napoli, Italy

eg Universita' di Napoli Federico II - Dipartimento di Fisica "Ettore Pancini", Italy

${ }^{e h}$ INAF - Istituto di Astrofisica Spaziale e Fisica Cosmica di Palermo, Italy

${ }^{e i}$ Istituto Nazionale di Fisica Nucleare - Sezione di Roma Tor Vergata, Italy

${ }^{e j}$ Universita' di Roma Tor Vergata - Dipartimento di Fisica, Roma, Italy

${ }^{e k}$ Istituto Nazionale di Fisica Nucleare - Sezione di Torino, Italy

${ }^{e l}$ Dipartimento di Fisica, Universita' di Torino, Italy

em Osservatorio Astrofisico di Torino, Istituto Nazionale di Astrofisica, Italy

en Uninettuno University, Rome, Italy

$f$ a Chiba University, Chiba, Japan

$f b$ National Institutes for Quantum and Radiological Science and Technology (QST), Chiba, Japan

$f c$ Kindai University, Higashi-Osaka, Japan

$f d$ Kanazawa University, Kanazawa, Japan 
$f e$ Institute for Cosmic Ray Research, University of Tokyo, Kashiwa, Japan

$f f$ Konan University, Kobe, Japan

$f g$ Kyoto University, Kyoto, Japan

$f^{h}$ Shinshu University, Nagano, Japan

${ }^{f i}$ National Astronomical Observatory, Mitaka, Japan

$f j$ Nagoya University, Nagoya, Japan

${ }^{f k}$ Institute for Space-Earth Environmental Research, Nagoya University, Nagoya, Japan

${ }^{f l}$ Graduate School of Science, Osaka City University, Japan

${ }^{m} m$ Institute of Space and Astronautical Science/JAXA, Sagamihara, Japan

${ }^{f n}$ Saitama University, Saitama, Japan

fo Hokkaido University, Sapporo, Japan

$f p$ Osaka Electro-Communication University, Neyagawa, Japan

$f q$ Nihon University Chiyoda, Tokyo, Japan

$f r$ University of Tokyo, Tokyo, Japan

$f s$ High Energy Accelerator Research Organization (KEK), Tsukuba, Japan

${ }^{f t}$ RIKEN, Wako, Japan

$f u$ Now at ESA, Holland

ga Korea Astronomy and Space Science Institute (KASI), Daejeon, Republic of Korea

${ }^{b}$ Sungkyunkwan University, Seoul, Republic of Korea

ha Universidad Nacional Autónoma de México (UNAM), Mexico

$h b$ Universidad Michoacana de San Nicolas de Hidalgo (UMSNH), Morelia, Mexico

$h c$ Benemérita Universidad Autónoma de Puebla (BUAP), Mexico

${ }^{h d}$ Universidad Autónoma de Chiapas (UNACH), Chiapas, Mexico

he Centro Mesoamericano de Física Teórica (MCTP), Mexico

ia National Centre for Nuclear Research, Lodz, Poland

${ }^{i b}$ Faculty of Physics, University of Warsaw, Poland

${ }^{j a}$ Institute of Space Science ISS, Magurele, Romania

$k a$ Joint Institute for Nuclear Research, Dubna, Russia

$k b$ Skobeltsyn Institute of Nuclear Physics, Lomonosov Moscow State University, Russia

$k c$ Space Regatta Consortium, Korolev, Russia

${ }^{l a}$ Institute of Experimental Physics, Kosice, Slovakia

$l b$ Technical University Kosice (TUKE), Kosice, Slovakia

$m a$ Universidad de León (ULE), León, Spain

$m b$ Instituto Nacional de Técnica Aeroespacial (INTA), Madrid, Spain

${ }^{m c}$ Universidad de Alcalá (UAH), Madrid, Spain

$m d$ Universidad Politécnia de madrid (UPM), Madrid, Spain

me Instituto de Astrofísica de Canarias (IAC), Tenerife, Spain

${ }^{n a}$ KTH Royal Institute of Technology, Stockholm, Sweden

${ }^{o a}$ Swiss Center for Electronics and Microtechnology (CSEM), Neuchâtel, Switzerland

$o b$ ISDC Data Centre for Astrophysics, Versoix, Switzerland

$o c$ Institute for Atmospheric and Climate Science, ETH Zürich, Switzerland

pa Space Science Laboratory, University of California, Berkeley, CA, USA

${ }^{p b}$ University of Chicago, IL, USA 
${ }^{p c}$ Colorado School of Mines, Golden, CO, USA

$p d$ University of Alabama in Huntsville, Huntsville, AL; USA

$p e$ Lehman College, City University of New York (CUNY), NY, USA

$p f$ NASA Marshall Space Flight Center, Huntsville, AL, USA

$p g$ University of Utah, Salt Lake City, UT, USA

$p h$ Georgia Institute of Technology, USA

${ }^{p i}$ University of Iowa, Iowa City, IA, USA

$p j$ NASA Goddard Space Flight Center, Greenbelt, MD, USA

${ }^{p k}$ Center for Space Science \& Technology, University of Maryland, Baltimore County, Baltimore, MD, USA

${ }^{p l}$ Department of Astronomy, University of Maryland, College Park, MD, USA

${ }^{p m}$ Fairfield University, Fairfield, CT, USA 